\title{
Surface Texture Synthesis and Mixing Using Differential Colors
}

\author{
Qing Wu \\ Lin Shi \\ Stephen Bond \\ University of Illinois at Urbana-Champaign
Email: $\{$ linshi,qingwu1,sdbond,yyz $@ @$ uiuc.edu
}

Yizhou Yu

\begin{abstract}
In neighborhood-based texture synthesis, adjacent local regions need to satisfy color continuity constraints in order to avoid visible seams. Such continuity constraints seriously restrict the variability of synthesized textures, making it impossible to generate new textures by mixing multiple input textures with very different base colors. In this paper, we propose to relax such restrictions and decompose synthesis into two relatively disjoint stages. In the first stage, an intermediate synthesized texture is generated by only considering the high frequency details during region search and matching. Such a scheme broadens the search space during texture synthesis, but may produce obvious seams due to large discontinuities in low frequency components. In the second stage, instead of performing local feathering along these discontinuities, we perform Laplacian texture reconstruction, which retains the high frequency details but computes new consistent low frequency components to eliminate the seams. It does not only affect texels close to the discontinuities, but also modifies the rest of the texels. Therefore, it can be viewed as a global feature-preserving smoothing step, and is more effective than local feathering. Experiments indicate that our twostage synthesis can produce desirable results for regular texture synthesis as well as texture mixing from multiple sources.
\end{abstract}

\section{INTRODUCTION}

Texture synthesis has been widely recognized as an important research topic in computer graphics. Early texture synthesis algorithms were based on global statistical models. Instead of enforcing global statistics, preserving the local arrangement of pixels has proven to be more effective in terms of visual quality. This intuition led to the neighborhood-based search-and-copy algorithms. Given a small texture example, these algorithms can produce larger textures that have similar texture elements and structures as the given example. Every output texture from such neighborhood-based texture synthesis is essentially a spatial rearrangement of the original local regions in the given example. When there is only a single small texture example, the number of possible rearrangements is actually limited because adjacent local regions need to satisfy continuity restrictions.

We propose to relax such neighborhood-based texture synthesis along two directions to improve the variability of the synthesized results without compromising their visual quality. First, relax the continuity restrictions. Previously, adjacent local regions in the output texture are typically required to have pixelwise color similarity in their overlapping portion. When a new local region needs to be chosen from the texture example, such a stringent condition results in a very small number of candidates and quite often zero candidates. We suggest to relax such region matching by focusing on the high frequency components only and overlooking the average color and intensity. Indeed, it is the high frequency components that play the most important role in characterizing a texture.

Second, allow multiple input texture examples. Sampling local regions from a single small texture example can only produce very limited variability. Ideally, example-based synthesis should generate results by sampling a large database. However, different textures may be acquired by different imaging devices and/or settings, under different illumination conditions, etc. Even the same real-world texture, such as grass, can appear very different in different texture images. Effectively sampling, matching and mixing local regions from multiple texture examples simultaneously is nontrivial.

In this paper, we focus on surface texture synthesis and propose a novel two-stage synthesis approach to accommodate these two relaxations. In the first stage, an intermediate synthesized texture is generated by only considering the high frequency details during region search and matching. It is achieved by using both features and rectified versions of the input textures. Each pixel value in the rectified textures is defined by its original value normalized by the accumulated intensity within a neighborhood. Such a scheme broadens the search space during texture synthesis. It facilitates sampling local regions from different texture examples as well as placing regions with very different average colors and intensities next to each other in the output texture. However, this intermediate texture has obvious seams due to large discontinuities in low frequency components.

In the second stage, to seamlessly mix local regions together and create smooth transitions among them, we perform texture reconstruction using differential colors, which is in the same spirit of Poisson image editing [1] and related surface editing [2]. It retains the high frequency details, but computes new consistent low frequency components for all local regions so that the seams among them disappear. Therefore, it can be viewed as a global feature-preserving smoothing step, and is more effective than local feathering. The Laplacian operator extracts local differential quantities which represent high frequency texture details. Given the Laplacian of the intermediate texture, we set up a sparse linear system with the new texture colors as the unknowns. The solution of this system not only retains the original texture details, but also provides a consistent coloring to all the texels in the synthesized texture without discontinuities along the boundaries of adjacent regions. 


\section{A. Related Work}

This paper is partially inspired by the recent success of texture synthesis [3], [4], [5], [6], [7], [8], [9], [10], [11], [12]. 2D textures are frequently modeled as Markov Random Fields, which give rise to the neighborhood based texture synthesis algorithms. Some algorithms model textures as a set of features, and generate new images by matching feature statistics, such as histograms and co-occurrences, potentially across multiple resolutions [3]. Patch-based texture synthesis [4], [5], [7], [8] achieved better results than earlier methods in terms of both quality and efficiency. Feature continuity on the boundary of two adjacent patches is an important issue and [5], [7], [8] have attempted to alleviate this problem using dynamic programming, graph cuts, and feature maps, respectively. Further improvements include real-time parallel synthesis on GPUs [9] and multiscale synthesis [10]. Recently, techniques have been developed to support the synthesis of textures with multiple layers using either signed distance functions or levelsets [11], [12].

There has also been much work [13], [14], [15], [16], [17], [18] on generalizing 2D texture synthesis onto meshes with arbitrary topology. Neighborhood based 2D synthesis was generalized to meshes in [13], [14], which perform hierarchical vertex-based synthesis. A binary texton mask was introduced in [16] as guidance data to improve synthesis results and reduce the number of broken features. Patch-based synthesis has also been generalized to meshes [15], [17]. A hierarchical patch-based approach was proposed in [15], and an efficient synthesis method on triangle meshes was introduced in [17] . A very fast synthesis technique accelerated by precomputed Jump Maps was introduced in [18]. Neighborhood or patch matching in these methods is directly based on color differences instead of differences in high frequency components.

Meanwhile, researchers have synthesized textures from multiple input examples by mixing together different elements from them [3], [16]. In [3], statistical learning trees are used to mix textures. On the other hand, the technique in [16] focuses on creating a progressive transition between different texture elements. However, these texture mixing techniques cannot globally adjust the colors of the mixed textures to make them more consistent with each other.

\section{OVERVIEW}

The input to our algorithm is a triangle mesh as well as one or more texture examples. The output is the same mesh covered with a texture synthesized from the given examples. In the case of multiple input textures, the synthesized texture is a spatial mixture of the texture elements from the texture examples. steps.

Our Laplacian texture synthesis algorithm has three basic

1) Apply a revised version of the method in [17] to generate an initial texture patch assignment on the mesh. The method in [17] does not directly synthesize textures on a mesh. Instead, it assigns a triangular texture patch in the input textures to each triangle in the mesh. The assigned texture patches of two adjacent triangles have a certain degree of continuity along their shared edge. Our revised version tries to emphasize high frequency details, but overlook the differences in the average colors of local regions. At the end of this step, each triangle in the mesh is associated with three pairs of texture coordinates which record the locations of the corners of its corresponding triangular texture patch in the input texture examples.

2) Each triangle in the mesh is tesselated with a highresolution grid and the assigned texture patch of the triangle is resampled onto this grid. A graphcut algorithm is further executed to refine the boundary between two adjacent texture patches so that such a boundary is not necessarily coincidental with the shared edge between two adjacent triangles any more. As a result, the transitions of details among texture patches are improved though their average colors may still be quite different.

3) Laplacian texture reconstruction is performed simultaneously on all the resampled texture patches from the previous step to eliminate the color discontinuities between adjacent patches. The Laplacian at each grid point is obtained from the original colors in the input texture examples.

\section{INITIAL TEXTURE ASSIGNMENT}

Our initial texture assignment is based on the method in [17], where a texton is defined to be a distinct local texture neighborhood. By clustering all neighborhoods with a fixed size from the given texture example, a small collection of textons can be extracted. They are the representatives of the clusters. During synthesis, triangular texture patches are grown on the mesh one by one to cover all the triangles. Note that each triangle in the mesh shares an edge with at most three adjacent triangles. During each step of synthesis, this method focuses on one triangle and counts the number of its adjacent triangles that have been covered with texture. If none of them has been covered, the current triangle is a seed and should be covered with a random patch. If one of them has been covered, we need to search for a texture patch in the given texture example that agrees well with the texture on this adjacent patch, which means that the two texton sequences on the shared edge should be similar. This strategy can be easily generalized to cases where two or three adjacent triangles are already covered with textures.

To emphasize high frequency details but overlook differences in average intensity and color, in our revised version of this method, we utilize a rectified version of each input texture. The rectified version of a texture is a greyscale image with a normalized intensity value at each pixel. We set the initial greyscale value at a pixel to be its luminance value which is a weighted average of the original three color channels. Suppose we define an $N \times N$ neighborhood for each pixel and the luminance at a neighbor $(i, j)$ is $I_{i j}$. The luminance value at each pixel is further normalized by the accumulated luminance in its neighborhood, which is $\left(\sum_{i j} I_{i j}^{2}\right)^{1 / 2}$. In this rectified texture, we essentially have removed the low frequency components, but retained the important high frequency details. We only use greyscale values because they are weighted averages of three color channels and contain high frequency details from all of them. In practice, using greyscale values indeed can 


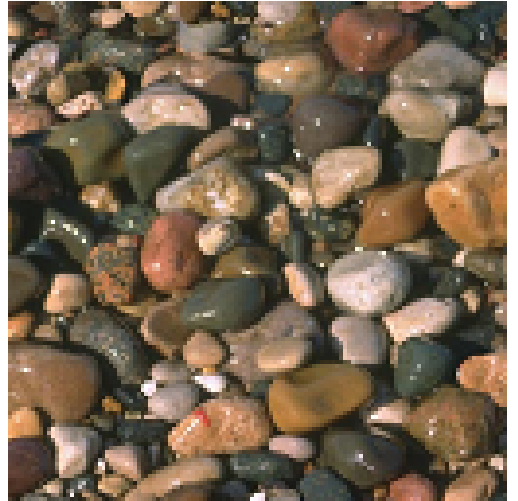

(a)

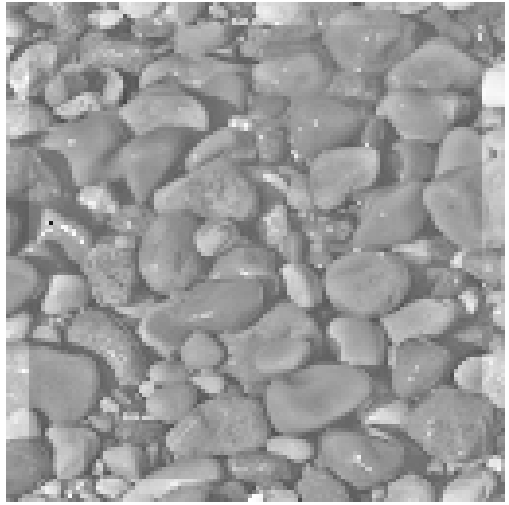

(b)

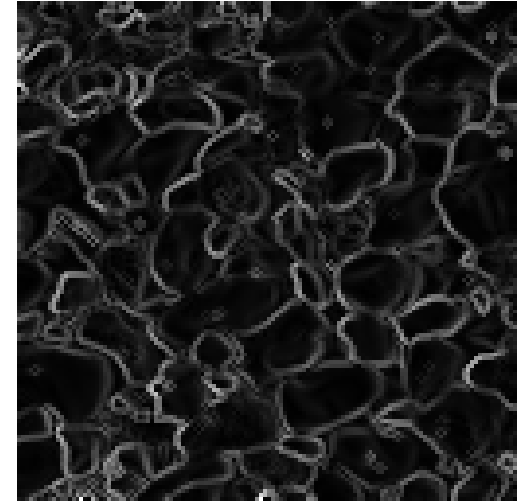

(c)

Fig. 1. (a) The original PEBBLES texture. (b) The rectified greyscale image of (a). (c) A feature image of (a) obtained by filtering.

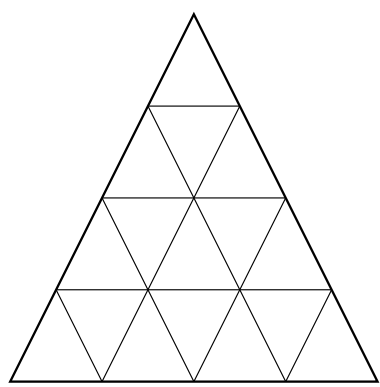

(a)

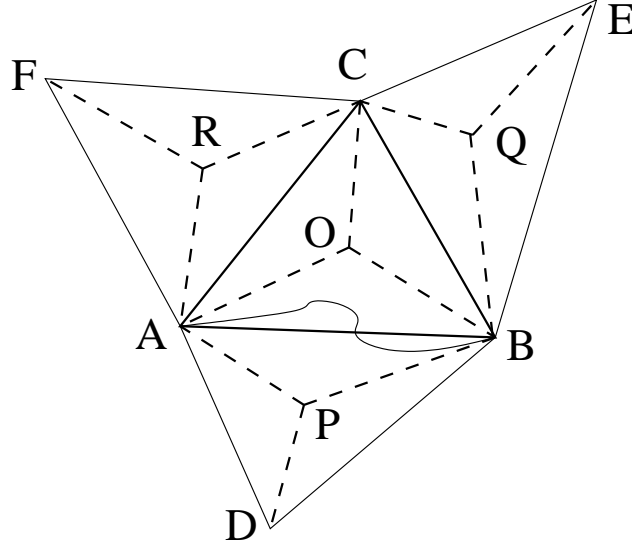

(b)

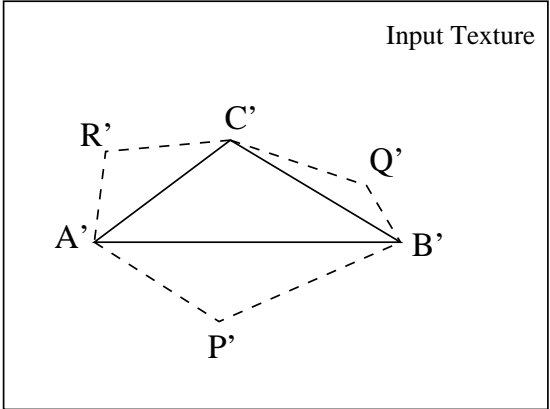

(c)

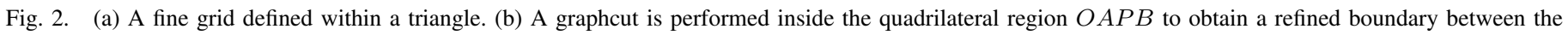
two adjacent texture patches. (c) An extended hexagonal texture patch corresponding to $\triangle A B C$ in (b).

produce better matching results than using three independent color channels. An example of a rectified texture is shown in Fig. 1(b). In practice, we set the size of the neighborhoods to be $11 \times 11$.

In addition to the rectified textures, we also obtain a feature image for each of the input texture examples. We first apply bilateral filtering [19] to remove noise while preserving features. In the bilateral filter, the scale of the closeness function $\sigma_{d}$ is set to 2.0, and the scale of the similarity function $\sigma_{r}$ is set to 10 out of 256 greyscale levels. We then use finite differences along the two image axes as a simple gradient estimator to obtain an edge response at every pixel. The pixelwise gradient estimation is used to form the feature images. An example of a feature image is shown in Fig. 1(c).

In our revised version of the method in [17], we use these rectified textures along with the feature images as the input to texton clustering. Thus, the neighborhood corresponding to each texton has a normalized greyscale pattern and a feature pattern. Both patterns emphasize high frequency details. In practice, weighted versions of these patterns are used for texton clustering. The weight for the greyscale pattern is set to 1.0, and the weight for the feature pattern is set to 0.3. These weighted patterns are treated as different channels of the same texture neighborhood during texton clustering. Once we have the collection of textons, the rest of the synthesis steps follow [17].

When there are multiple input textures, every time we need to search for a texture patch for a triangle, we find the best candidate from each input texture and then choose among them the one with the highest matching score. Usually, we would like to set up for each input texture a target percentage in the output texture. To approximately control the synthesis process using these target percentages, we define a Gaussian function for each input. The standard deviation of the Gaussian is set to be the target percentage of the input texture. The function value of the Gaussian is used to modulate the matching score. When the actual percentage is lower than the target percentage, the Gaussian returns a large value which does not obviously affect the matching score; when the actual percentage is higher than the target percentage, the Gaussian returns a small value which significantly decreases the matching score. Thus, these Gaussian functions implicitly control the likelihood of sampling a specific input texture.

\section{TEXTURE RESAMPLING AND BOUNDARY REFINEMENT}

Since we would like to perform boundary refinement on the triangular texture patches and Laplacian reconstruction over 


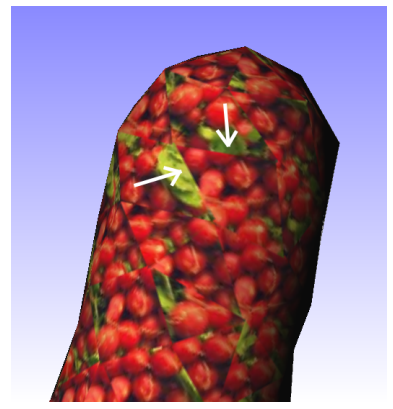

(a)

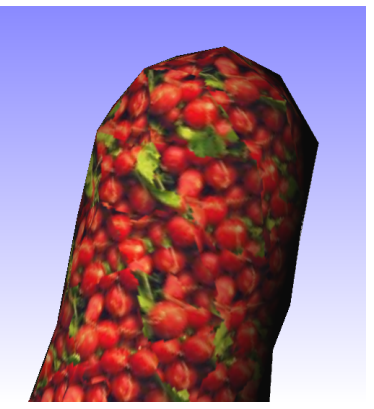

(b)
Fig. 3. (a) Initial texture patch assignment result with two of the seams indicated by arrows. (b) Result obtained from boundary refinement with Graphcut.

the entire synthesized texture, indexing the texture patch for each triangle as three pairs of texture coordinates in the input texture space becomes insufficient. To facilitate these later steps, we resample the texture patches onto a high-resolution grid over the original mesh surface. The high-resolution grid within each triangle is set up using barycentric coordinates as shown in Fig. 2(a). That is, every edge of the triangle has the same number of sample points. We actually further enforce that all edges in the triangle mesh have the same number of sample points. Thus, the subgrids within two adjacent triangles coincide on their shared edge to avoid T-junctions. If the original triangle mesh has some overly large or elongated triangles, we split those triangles in a preprocessing step while avoiding T-junctions.

We resample the texture patches previously assigned to the triangles onto this high-resolution grid. As shown in Fig. 2(b)(c), suppose a triangle $\triangle A B C$ in the mesh has a corresponding triangular texture patch $\Delta A^{\prime} B^{\prime} C^{\prime}$ in one of the input texture examples. To facilitate boundary refinement at a later step, we actually resample an area larger than $\triangle A^{\prime} B^{\prime} C^{\prime}$. Suppose $\triangle A B D, \triangle B C E$ and $\triangle C A F$ are the three triangles adjacent to $\triangle A B C$. Their centers are $P, Q$ and $R$, respectively. We first flatten these three triangles onto the same plane where $\triangle A B C$ resides and obtain the new locations of their centers. From these new locations, we can further obtain their corresponding locations $P^{\prime}, Q^{\prime}$ and $R^{\prime}$ in the 2D texture space. We resample the entire hexagonal area $A^{\prime} P^{\prime} B^{\prime} Q^{\prime} C^{\prime} R^{\prime}$ in the texture space onto the corresponding region, $A P B Q C R$, of the high-resolution grid. Thus, each resampled texture patch of a triangle extends into its three adjacent triangles. Suppose the center of $\triangle A B C$ is $O$. During this resampling, $\triangle O A B$, $\triangle O B C$ and $\triangle O C A$ not only obtain color values from the texture patch originally assigned to $\triangle A B C$, but also obtain a second color value from the extended hexagonal patches corresponding to the three adjacent triangles of $\triangle A B C$.

During initial texture patch assignment discussed in the previous section, each triangle is assigned a triangular texture patch. The boundary between two adjacent texture patches coincide with the shared edge of their corresponding triangles. In a subsequent boundary refinement procedure, we apply the graphcut algorithm in [7] to refine the boundaries between resampled texture patches on the high-resolution grid. We need to take into account the extended hexagonal texture patches to perform this procedure. Consider triangles $\triangle A B C$ and $\triangle A B D$ in Fig. 2(b). Suppose their hexagonal texture patches are $H T P_{O}$ and $H T P_{P}$, respectively. These two texture patches have an overlapping quadrilateral region, $O A P B$. We set up a minimum graph cut problem as follows. The grid points closest to $O A$ and $O B$ are constrained to have colors from the texture patch $H T P_{O}$ while the grid points closest to $P A$ and $P B$ are constrained to have colors from the patch $H T P_{P}$. The vertices $A$ and $B$ also have fixed colors. We then seek a minimum graph cut between $A$ and $B$ and within the region $O A P B$. The algorithm in [7] is applied to find this cut which can provide a better transition between the high frequency details of the two texture patches than the original boundary. The grid points falling on the same side of the cut as $O$ obtain colors from patch $H T P_{O}$ while the grid points on the other side of the cut obtain colors from patch $H T P_{P}$. Note that we still use the rectified textures during boundary refinement because the original texture colors may have large discontinuities along the triangle edges, which prevent the graphcut algorithm to find a different cut that provides better transition for high frequency details. Fig. 3(a)-(b) demonstrate the effectiveness of this boundary refinement procedure.

Since the colors of the mesh vertices are not refined at all during the aforementioned boundary refinement, we also designed another graphcut procedure specifically tailored for them. We first define an umbrella region centered at each vertex, and then flatten that region onto a parameterization plane. A subsequent graph cut is performed in this flattened region to refine the boundaries of the texture patches there. However, in our experiments, we have not observed any obvious improvements in visual quality due to this vertexcentric refinement. Therefore, we leave it as an optional step.

\section{LAPLACIAN TEXTURE ReCONSTRUCTION}

The synthesis process in the previous sections focuses on high frequency details. We call the surface texture synthesized by the previous steps the intermediate texture. There are obvious seams inbetween adjacent texture patches in the intermediate texture because of discontinuities in the low frequency components. To remove these large discontinuities while still preserving high frequency texture details, we present a texture reconstruction technique based on the Laplacian operator which encodes high frequency features. Given the estimated Laplacians of the intermediate texture, the reconstruction process tries to obtain a new continuous surface texture which can reproduce the Laplacians. The reconstruction process uses the high-resolution grid previously generated for texture resampling.

\section{A. A Weighted Laplacian Operator}

The Laplacian of a vertex $\mathbf{v}_{i}$ in the high-resolution grid is computed by collecting the colors of its 1-ring neighbors as shown in Fig. 4. To compensate the non-uniform shape of the triangles, Fujiwara weights [20] are used:

$$
L\left(\mathbf{v}_{i}\right)=-\sum_{0 \leq j<N(i)} \frac{1}{e_{i_{j}}}\left(c_{i}-c_{i_{j}}\right),
$$

where $\mathbf{v}_{i_{j}}$ is a vertex directly connected to $\mathbf{v}_{i}, e_{i_{j}}$ represents the edge length between $\mathbf{v}_{i}$ and $\mathbf{v}_{i_{j}}, c_{i}$ and $c_{i_{j}}$ represent the colors at the vertices, and $N(i)$ represents the number of 


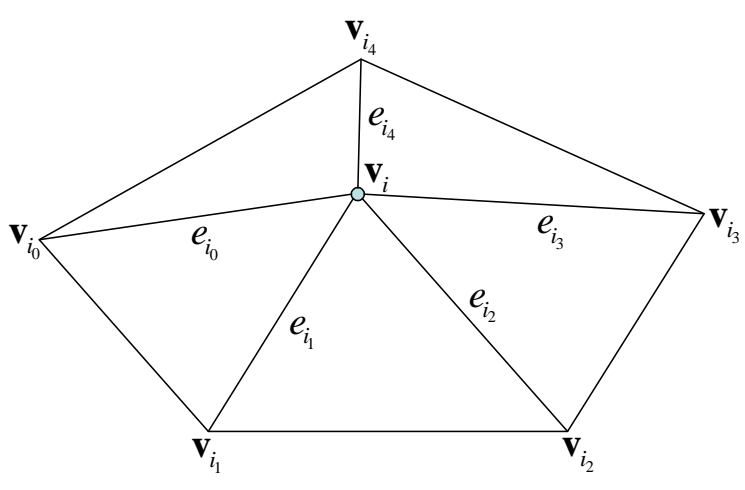

Fig. 4. The 1-ring structure of a vertex, $\mathbf{v}_{i}$.

neighboring vertices of $\mathbf{v}_{i}$. This Laplacian operator can also be rewritten as:

$$
L\left(\mathbf{v}_{i}\right)=-\sum_{0 \leq j<N(i)} L^{f}\left(\mathbf{v}_{i}, \mathbf{v}_{i_{j}}, \mathbf{v}_{i_{j+1}}\right),
$$

where $L^{f}\left(\mathbf{v}_{i}, \mathbf{v}_{i_{j}}, \mathbf{v}_{i_{j+1}}\right)=\frac{1}{2 e_{i_{j}}}\left(c_{i}-c_{i_{j}}\right)+\frac{1}{2 e_{i_{j+1}}}\left(c_{i}-c_{i_{j+1}}\right)$. $L^{f}\left(\mathbf{v}_{i}, \mathbf{v}_{i_{j}}, \mathbf{v}_{i_{j+1}}\right)$ only involves the three vertices of a triangular face in the 1-ring structure of $\mathbf{v}_{i}$. This formulation allows us to consider the Laplacian operator as a summation of these facewise terms.

Since we would like to remove discontinuities along patch boundaries but still preserve original high frequency features within the texture patches, it is desirable to have spatially adaptive smoothing. To achieve this goal, we designed a weighted Laplacian operator which imposes potentially different weights on the edges. Eqs. (1) and (2) thus become

$$
\begin{array}{r}
L_{w}\left(\mathbf{v}_{i}\right)=-\sum_{0 \leq j<N(i)} \frac{w_{i_{j}}}{e_{i_{j}}}\left(c_{i}-c_{i_{j}}\right) \\
=-\sum_{0 \leq j<N(i)} L_{w}^{f}\left(\mathbf{v}_{i}, \mathbf{v}_{i_{j}}, \mathbf{v}_{i_{j+1}}\right),
\end{array}
$$

where $w_{i_{j}}$ is a positive weight for the edge between $\mathbf{v}_{i}$ and $\mathbf{v}_{i_{j}}$, and $L_{w}^{f}\left(\mathbf{v}_{i}, \mathbf{v}_{i_{j}}, \mathbf{v}_{i_{j+1}}\right)$ is also a facewise term similar to $L^{f}\left(\mathbf{v}_{i}, \mathbf{v}_{i_{j}}, \mathbf{v}_{i_{j+1}}\right)$. If both $\mathbf{v}_{i}$ and $\mathbf{v}_{i_{j}}$ are from the same texture patch, we simply set $w_{i_{j}}=1$; otherwise, $w_{i_{j}}$ can be either smaller or larger than 1 . If the weight of an edge is less than 1 , the bonding between the two vertices of the edge is weakened, and there is less smoothing across the edge. Too small a weight may increase the stiffness of the resulting linear system discussed in the next section.

\section{B. Laplacian Reconstruction}

Given the Laplacians of the intermediate texture, we would like to reconstruct a new texture with the same Laplacians. Therefore, we set up a linear system with one equation per vertex. The equation for vertex $\mathbf{v}_{i}$ is expressed as

$$
-\sum_{0 \leq j<N(i)}\left(\frac{w_{i_{j}}}{2 e_{i_{j}}}\left(c_{i}-c_{i_{j}}\right)+\frac{w_{i_{j+1}}}{2 e_{i_{j+1}}}\left(c_{i}-c_{i_{j+1}}\right)\right)=L_{i},
$$

where $c_{i}$ and $c_{i_{j}}$ represent unknown vertex colors in the new texture we would like to solve and $L_{i}$ represents the estimated Laplacian of the intermediate texture at $\mathbf{v}_{i}$ using Eq. (3). The left hand side of this equation is actually the weighted Laplacian of the unknown new texture at $\mathbf{v}_{i}$. Since the weighted Laplacian is a linear operator, this equation is a linear equation of the unknown vertex colors. Note that if the textures have three color channels, there are three equations for each vertex. The collection of equations for all the vertices form a sparse linear system which has a symmetric coefficient matrix. Since the Laplacian operator is translation invariant, we need to fix the color of at least one vertex in order to obtain a unique solution of the linear system. Such fixed colors essentially form a boundary condition of the equations. Efficient iterative solvers [21] are a good choice for such a sparse linear system. In practice, we use a preconditioned (Incomplete Choleskey Factorization) conjugate gradient method [22].

1) Laplacian Estimation at Patch Boundaries: There are additional details concerning the estimation of the right hand side of Eq. (5) since the intermediate texture consists of patches with discontinuities on their boundaries. According to Eq. (3), the weighted Laplacian of a vertex can be estimated by accumulating a simpler term, $L_{w}^{f}\left(\mathbf{v}_{i}, \mathbf{v}_{i_{j}}, \mathbf{v}_{i_{j+1}}\right)$, over all the triangular faces surrounding the vertex. However, a triangle may stride two or more texture patches. We summarize the estimation of this term as follows.

- If $\mathbf{v}_{i}, \mathbf{v}_{i_{j}}$ and $\mathbf{v}_{i_{j+1}}$ belong to the same patch, we directly use their colors to estimate $L_{w}^{f}\left(\mathbf{v}_{i}, \mathbf{v}_{i_{j}}, \mathbf{v}_{i_{j+1}}\right)$.

- If the three vertices belong to two different patches, we should not directly use their existing colors because there may be a large gap among them. Since there must be a dominant patch having two of the three vertices, during the estimation of $L_{w}^{f}\left(\mathbf{v}_{i}, \mathbf{v}_{i_{j}}, \mathbf{v}_{i_{j+1}}\right)$, the color of the third vertex should be taken from an extended version of the dominant patch to avoid large gaps.

- If the three vertices belong to three different patches, we simply randomly choose a dominant patch from the three. The colors of the other two vertices are taken from an extended version of that dominant patch.

2) Global Reconstruction: When the average brightness and color of the patches in the intermediate texture differ significantly (e.g. when they are from different complicated textures), we set up a sparse boundary condition and simultaneously solve the system of equations in (5) to remove the differences. As mentioned earlier, the boundary condition should consist of at least one constraint on the variables. A simple equality constraint is declared by setting the color of a vertex to be a fixed value. Such constraints reduce the number of variables in the linear system. The reduced linear system has a unique solution. The user can choose to interactively specify such constraints. In the absence of user-defined constraints, our program chooses to fix the colors at the centers of a random subset of the patches in the mesh. A more sophisticated constraint is defined by setting a linear combination of the vertex colors to be a fixed value. For example, we experimented with setting the average of all vertex colors to be a fixed value. Such linear constraints can be integrated into the linear system by considering them as additional equations. When there is exactly one linear constraint, the resulting enhanced linear system has a unique solution which defines a globally continuous new surface texture. When there is more than one 
linear constraints, the system becomes overdetermined, and a least-squares solution should be obtained.

3) Local Reconstruction: When the average brightness and color of the patches in the intermediate texture are similar (e.g. when they are all from the same sample texture), locally reconstructing the texture can produce good results with much less computational cost than the global reconstruction. This is done by imposing color constraints on all the boundary vertices of the texture patches. The constrained color for every boundary vertex $\mathbf{v}_{i}$ is computed by simply blending the existing colors in its 1-ring structure,

$$
c_{\text {fixed }}\left(\mathbf{v}_{i}\right)=\frac{1}{N(i)} \sum_{0 \leq j<N(i)} c_{i_{j}},
$$

where the neighboring vertices of $\mathbf{v}_{i},\left\{\mathbf{v}_{i_{j}}\right\}$, may belong to different patches. These dense color constraints effectively disconnect the texture patches from each other. The resulting linear system can be solved patch by patch. In practice, this scheme is a few times faster than the global reconstruction. Nevertheless, it only propagates information within each texture patch, which makes it better than local feathering along the patch boundaries but prevents it from resolving color differences on patches that are remote to each other. Therefore, this local scheme provides a tradeoff between quality and efficiency.

Fig. 5 demonstrates the visual quality of both local and global texture reconstruction. In the intermediate textures, there are obvious seams among the patches due to differences in low frequency components. Local Laplacian reconstruction can certainly remove these seams and create smooth transitions among the patches. However, it fails to produce large-scale changes that would make the base colors of the patches more consistent. On the other hand, global Laplacian reconstruction can perform such large-scale changes and produce more desirable results. To fully test the capability of global reconstruction, in the last example shown in Fig. 5, we artificially add a large random color shift to every texture patch in the intermediate texture. Global reconstruction can successfully remove these large color shifts and recover a consistent base color for all the patches. The reconstructed surface texture appears similar to the original input texture.

\section{AdDitional ExPERIMENTAL RESUltS}

We have conducted a large number of experiments on surface texture synthesis and mixing using the algorithm developed in this paper. Besides the examples shown in Fig. 5, we show a few additional results in Fig. 6. The first example in Fig. 6 gives a good demonstration on the fact that our algorithm can significantly improve the variability of the synthesized textures. The original FLOWERS texture has too few color variations. Extending such a texture over a large surface area would not make the result very appealing. By mixing it with the two leaf textures, the synthesized results become more interesting. In the first row of Fig. 6, the left one is the local reconstruction result while the right one is the global reconstruction result. In this particular case, both of them look interesting. The local result retains the rich colors of the three input textures while the global result has a smooth and subtle color change over the entire mesh.
TABLE I. THE NUMBER OF VERTICES AND FACES IN THE MESHES AND THEIR CORRESPONDING FINE GRIDS USED IN OUR EXPERIMENTS.

\begin{tabular}{|c|c|c|}
\hline & \# mesh vertices/faces & \# grid vertices/faces \\
\hline \hline Bunny & $2503 / 5002$ & $640258 / 1280512$ \\
\hline Camel & $2444 / 4884$ & $625154 / 1250304$ \\
\hline Pawn & $510 / 1016$ & $130050 / 260096$ \\
\hline V-shape & $170 / 336$ & $43010 / 86016$ \\
\hline
\end{tabular}

TABLE II. THE AVERAGE RUNNING TIMES (IN SECONDS) OF DIFFERENT STAGES OF OUR ALGORITHM ON THREE MESHES. THESE TIMES WERE MEASURED ON A 3.2GHZ AMD PROCESSOR. "INITIAL" REFERS TO THE INITIAL TEXTURE PATCH ASSIGNMENT STAGE; "REFINEMENT" REFERS TO THE TEXTURE RESAMPLING AND BOUNDARY REFINEMENT STAGE; "LOCAL/GLOBAL LAPL" REFERS TO LOCAL AND GLOBAL LAPLACIAN TEXTURE RECONSTRUCTION.

\begin{tabular}{|c|c|c|c|}
\hline & Initial & Refinement & Local / Global Lapl \\
\hline \hline Bunny & 8 & 5 & $13 / 25$ \\
\hline Camel & 3 & 4 & $13 / 28$ \\
\hline Pawn & $<1$ & 1 & $2 / 4.5$ \\
\hline
\end{tabular}

The statistics of the meshes used in our experiments are summarized in Table I. The running times of various stages of our algorithm are also summarized in Table II.

\section{CONCLUSIONS}

In this paper, we proposed to decompose texture synthesis into two relatively disjoint stages. In the first stage, an intermediate synthesized texture is generated by only considering the high frequency details during neighborhood search and matching. In the second stage, we perform Laplacian texture reconstruction which retains the high frequency details but computes consistent low frequency components. It does not only affect texels close to discontinuities, but also modifies the rest of the texels. Therefore, it can be viewed as a global feature-preserving smoothing step, and is more effective than local feathering. Experiments indicate that our two-stage synthesis can produce desirable results for regular texture synthesis as well as texture mixing from multiple sources. In future, we would like to implement Laplacian reconstruction and other time-consuming steps on GPUs to achieve interactive performance.

\section{REFERENCES}

[1] P. Perez, M. Gangnet, and A. Blake, "Poisson image editing," $A C M$ Trans. on Graphics, vol. 22, no. 3, pp. 313-318, 2003.

[2] Y. Yu, K. Zhou, D. Xu, X. Shi, H. Bao, B. Guo, and H.-Y. Shum, "Mesh editing with poisson-based gradient field manipulation," ACM Trans. Graph., vol. 23, no. 3, pp. 644-651, 2004.

[3] Z. Bar-Joseph, R. El-Yaniv, D. Lischinski, and M. Werman, "Texture mixing and texture movie synthesis using statistical learning," IEEE Transactions on Visualization and Computer Graphics, vol. 7, no. 2, pp. 120-135, 2001.

[4] L. Liang, C. Liu, Y. Xu, B. Guo, and H.-Y. Shum, "Real-time texture synthesis using patch-based sampling," ACM Trans. Graphics, vol. 20, no. 3, pp. 127-150, 2001.

[5] A. Efros and W. Freeman, "Image quilting for texture synthesis and transfer," in SIGGRAPH'01, 2001, pp. 341-346.

[6] J. Dischler, K. Maritaud, B. Levy, and D. Ghazanfarpour, "Texture particles," Computer Graphics Forum, vol. 21, no. 3, pp. 401-410, 2002.

[7] V. Kwatra, A. Schödl, I. Essa, G. Turk, and A. Bobick, "Graphcut textures: Image and video synthesis using graph cuts," ACM Transactions on Graphics, vol. 22, no. 3, pp. 277-286, 2003. 

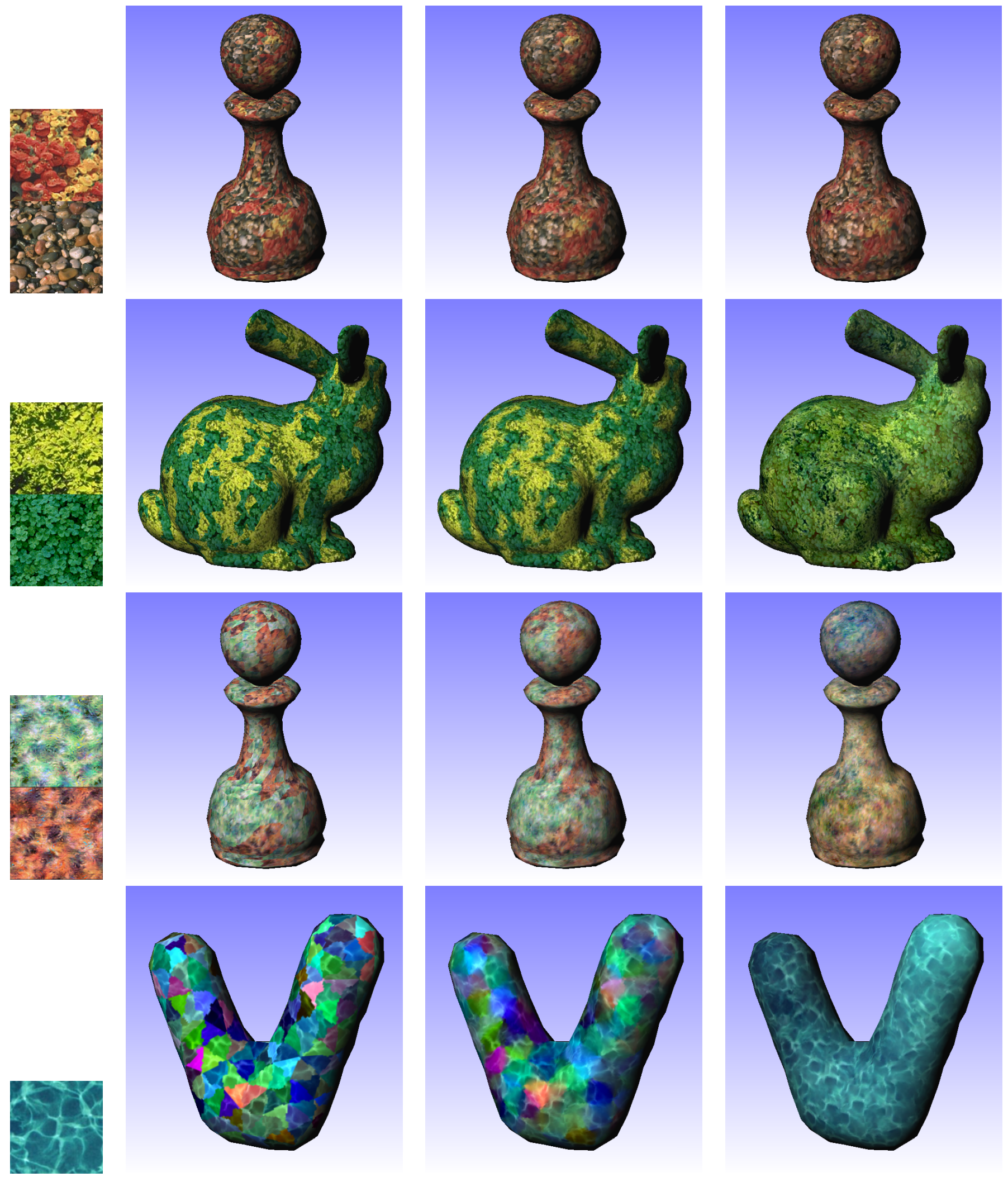

(a)

(b)

(c)

(d)

Fig. 5. (a) Input texture examples. (b) Synthesized intermediate textures with color discontinuities among patches. (c) Textures computed from local Laplacian reconstruction. (d) Textures computed from global Laplacian reconstruction. Note that local reconstruction works reasonably well for the texture mixture in the first row because the colors of the mixed texture patches are not too different. However, for the remaining three mixture examples, global reconstruction produces more natural and consistent low frequency components. The intermediate texture in the last row is artificially modified by adding a random color shift to each texture patch. Global texture reconstruction can successfully remove such color shifts. 

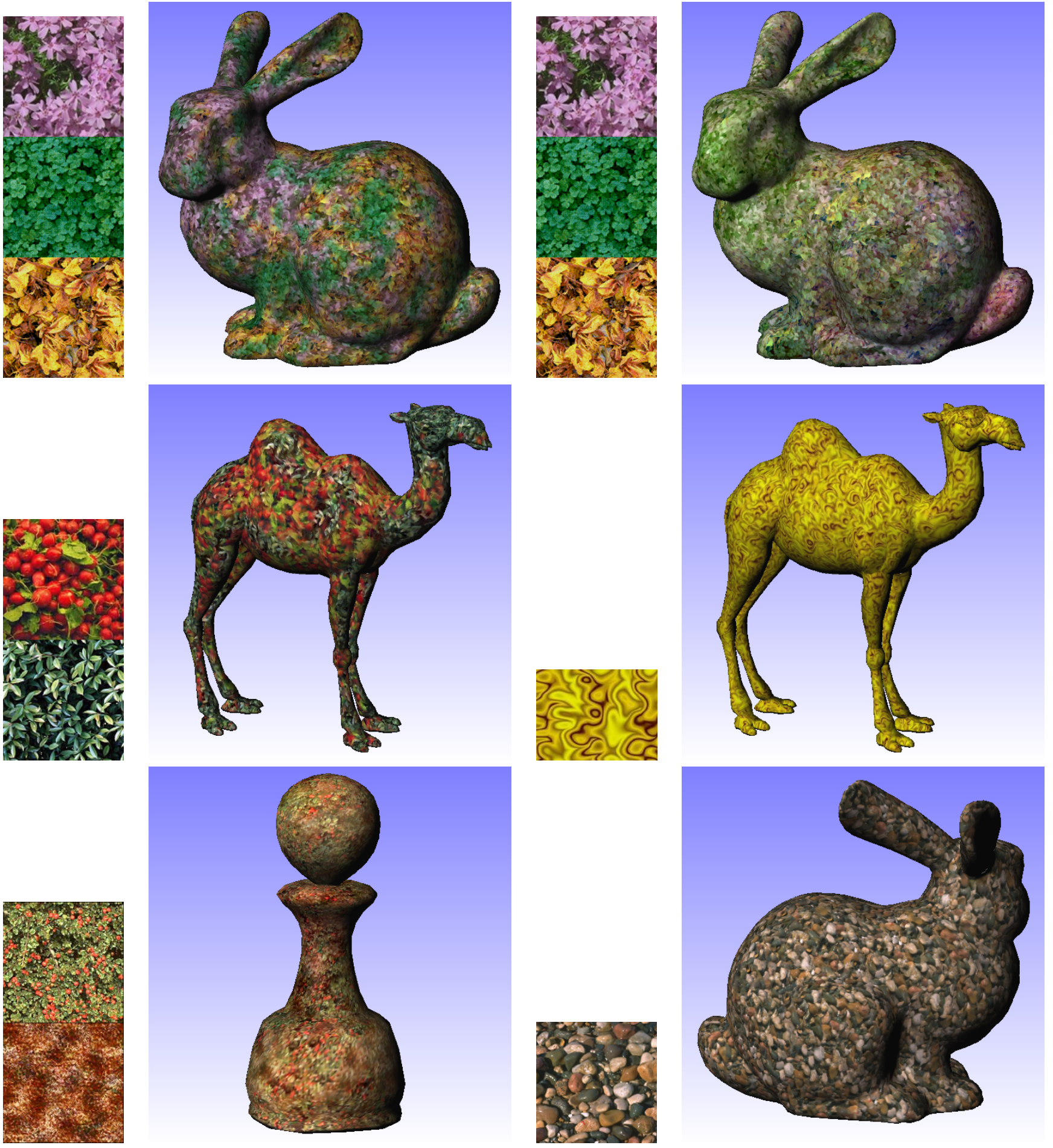

Fig. 6. Additional surface texture synthesis and mixing results.

[8] Q. Wu and Y. Yu, "Feature matching and deformation for texture synthesis," ACM Trans. Graph., vol. 23, no. 3, pp. 364-367, 2004.

[9] S. Lefebvre and H. Hoppe, "Parallel controllable texture synthesis," ACM Transactions on Graphics, vol. 24(3), pp. 777-786, 2005.

[10] C. Han, E. Risser, R. Ramamoorthi, and E. Grinspun, "Multiscale texture synthesis," ACM Transactions on Graphics, vol. 27(3), 2008.

[11] A. Rosenberger, D. Cohen-Or, and D. Lischinski, "Layered shape synthesis: automatic generation of control maps for non-stationary textures," in ACM Transactions on Graphics (TOG), vol. 28, no. 5.
ACM, 2009, p. 107.

[12] R. Wu, W. Wang, and Y. Yu, "Optimized synthesis of art patterns and layered textures," IEEE Transactions on Visualization and Computer Graphics, vol. 20, no. 3, pp. 436-446, 2014.

[13] G. Turk, "Texture synthesis on surfaces," in SIGGRAPH'01, 2001, pp. 347-354.

[14] L.-Y. Wei and M. Levoy, "Texture synthesis over arbitrary manifold surfaces," in SIGGRAPH'01, 2001, pp. 355-360.

[15] C. Soler, M.-P. Cani, and A. Angelidis, "Hierarchical pattern mapping," 
in SIGGRAPH'02, 2002, pp. 673-680.

[16] J. Zhang, K. Zhou, L. Velho, B. Guo, and H.-Y. Shum, "Synthesis of progressively-variant textures on arbitrary surfaces," in SIGGRAPH'03, 2003, pp. 295-302.

[17] S. Magda and D. Kriegman, "Fast texture synthesis on arbitrary meshes," in Eurographics Symposium on Rendering, 2003, pp. 82-89.

[18] S. Zelinka and M. Garland, "Jump map-based interactive texture synthesis," ACM Transactions on Graphics, vol. 23, no. 4, pp. 929-1073, 2004.

[19] C. Tomasi and R. Manduchi, "Bilateral filtering for gray and color images," in Proc. Intl. Conf. on Computer Vision, 1998, pp. 836-846.

[20] K. Fujiwara, "Eigenvalues of laplacians on a closed riemannian manifold and its nets," in Proceedings of the American Mathematical Society, 1995, pp. 123:2585-2594.

[21] Y. Saad, Iterative Methods for Sparse Linear Systems. PWS Publishing Company, 1996.

[22] D. Kershaw, "The incomplete cholesky-conjugate gradient method for the iterative solution of systems of linear equations," Journal of Computational Physics, vol. 26, no. 1, pp. 43-65, 1978. 